\title{
THE DIFFERENCE BETWEEN RADIO-LOUD AND RADIO-QUIET ACTIVE GALAXIES
}

\author{
A. S. WILSON \\ Space Telescope Science Institute \\ 3700 San Martin Drive, Baltimore, MD 21218 \\ and \\ Astronomy Department, University of Maryland, \\ College Park, MD 20742 \\ and \\ E. J. M. COLBERT \\ Astronomy Department, University of Maryland, \\ College Park, MD 20742
}




\begin{abstract}
The recent development of unified theories of active galactic nuclei (AGN) has indicated that there are two physically distinct classes of these objects - radio-loud and radio-quiet. The primary observational distinctions between the two types are: (1) The radio-loud objects produce large scale radio jets and lobes, with the kinetic power of the jets being a significant fraction of the total bolometric luminosity. On the other hand, the weak radio ejecta of the radio-quiet objects are energetically insignificant. (2) The radioloud objects are associated with elliptical galaxies which have undergone recent mergers, while the radio-quiets prefer spiral hosts. (3) The space density of the radio-louds at a given optical luminosity is $\approx 10$ times lower than that of the radio-quiets. Despite these differences, the (probably) thermal emissions from the AGN (continua and lines from Xray to infrared wavelengths) are quite similar in the two classes of object. We argue that this last result suggests that the black hole masses and mass accretion rates in the two classes are not greatly different, and that the difference between the classes is associated with the spin of the black hole.

We assume that the normal process of accretion through a disk does not lead to rapidly spinning holes, and propose instead that galaxies (e.g. spirals) which have not suffered a recent major merger event contain non-rotating or only slowly rotating black holes. When two such galaxies merge, the two black holes are known to form a binary and we assume that they eventually coalesce. In the small fraction of mergers in which the two "parent" galaxies contain very massive holes of roughly equal mass, a rapidly spinning, very massive hole results. It is proposed that such mergers are the progenitors of powerful radio sources, in which the radio jets are powered by the spin energy of the merged hole. We calculate the distributions of mass and spin for the merged holes from the parent hole mass distribution, which is derived from the optical luminosity function of radio-quiet AGN adopting different activity patterns. The ratio of the number of radio-loud to radio-quiet AGN's at a given thermal (e.g. optical) luminosity is determined by the galaxy merger rate. The required fraction of galaxies which merge during the average lifetime $\left(\approx 10^{8} \mathrm{yrs}\right)$ of a radio-loud
\end{abstract}


AGN is found to be $10^{-1}$, i.e. a merger rate of 1 in $\simeq 10^{9}$ yrs. The Blandford-Znajek formalism is then used to predict the radio luminosity and radio luminosity function of the merged population. Comparisons between the predicted and observed radio luminosity functions constrain the efficiencies with which jet power is extracted from the spinning hole and radio emission is produced by the jet. The cosmological evolution of the radio properties of the radio-loud objects is related to the increased frequency of merger events at earlier epochs.

Subject headings: black hole physics - galaxies: active - galaxies: interactions - galaxies: jets - galaxies: nuclei - galaxies: quasars: general 


\section{INTRODUCTION}

Over the past decade, a considerable unification in our understanding of the phenomenology of active galactic nuclei (AGN) has been achieved. Because radiation is not emitted isotropically by these nuclei, intrinsically similar types of AGN may appear quite different when viewed from different directions. Two kinds of anisotropy are recognised. One kind is emission in a narrow cone, which is almost certainly a result of beaming by a relativistic jet. The second kind of anisotropy involves emission in a wider cone, which is a consequence of either shadowing of an intrinsically isotropic, optical-ultraviolet source by a thick (or warped) dusty torus or an intrinsically anisotropic optical-ultraviolet emitter. Radio-quiet objects form one class of active galaxies, with Seyfert 1's and radio-quiet quasars viewed close to "pole-on" (so the broad line region is seen directly) and Seyfert 2 's viewed more equatorially (so the broad line region is hidden by the dusty torus). The second class comprises low power (Fanaroff-Riley class I, FRI) radio galaxies and BL Lac objects. A BL Lac is seen when the nucleus is viewed within the radiation beam of a relativistic jet which fuels the large-scale radio lobes. At larger viewing angles, the large-scale lobes dominate the radio emission and the object is a classical FRI radio galaxy. Lastly, OVV quasars, radio-loud quasars, broad-line and narrow-line radio galaxies (FRII) appear to form a third class, with the sequence representing increasing angular difference between our line of sight and the axis of the source. In this class, both kinds of anisotropy are believed to be present. These issues have been recently reviewed by Antonucci (1993).

The reason for the large range in radio powers is unknown. There has, however, been considerable discussion concerning whether the radio-quiet and radio-loud objects form distinct and separate classes, or whether there is a continuum of radio properties in just one class of object. Several arguments favor the former hypothesis, as we now summarise. (i) There is a difference in the nature of the host galaxies, at least at low redshift. Radio-loud AGN are never found in spiral hosts, but almost always reside in elliptical or elliptical-like galaxies (a few are associated with S0's). Conversely, relatively few radio-quiet AGN are found in ellipticals. (ii) Imaging surveys have shown that the mean absolute magnitudes 
of the underlying galaxies of radio galaxies and radio-loud quasars are the same $\left(<\mathrm{M}_{V}>\right.$ $=-23.3 ; \mathrm{H}_{0}=50 \mathrm{~km} \mathrm{~s}^{-1} \mathrm{Mpc}^{-1}$ and $\mathrm{q}_{0}=0$ are used throughout the paper), consistent with the notion that they are identical objects and that the observability of the quasar nucleus is determined by variability or aspect dependent effects (e.g. Malkan 1984; Smith et al. 1986; Véron-Cetty \& Woltjer 1990; see Hutchings 1987 for a contradictory result). In contrast, the host galaxies of radio-quiet quasars are 0.6 - $1.0 \mathrm{mag}$ less luminous (e.g. Smith et al. 1986; Véron-Cetty \& Woltjer 1990; see also review by Woltjer 1990). It has further been argued (e.g. Smith et al. 1986; Hutchings, Janson \& Neff 1989) that the host galaxies of radio-loud quasars are ellipticals and those of radio-quiet quasars are spirals, consistent with the distinction at low redshift. These results show that the radio-loud and radio-quiet classes inhabit different types of host galaxy. (iii) The radio luminosity functions of Seyfert galaxies and radio galaxies are distinct and do not join onto each other (Meurs \& Wilson 1984). Further, radio-loud AGN comprise an intrinsically rarer class of object than radio-quiets (e.g. Padovani 1993). (iv) Radio surveys of optically selected quasars commonly show a bimodal distribution of flux densities (e.g. Strittmatter et al. 1980; Kellermann et al. 1989). Peacock, Miller \& Longair (1986) favored the existence of two quasar populations with widely differing radio luminosities and differing optical luminosity functions. This conclusion was based on their finding that the radio properties of optically faint quasars conflicted with a single population model (Schmidt 1970) in which the optical and radio luminosities are correlated. In subsequent work, Miller, Peacock \& Mead (1990) performed a radio survey of optically selected quasars covering a wide range of optical luminosities, but only a narrow range of redshifts. These authors found strong evidence for the existence of two populations of quasar - one having radio luminosities $L_{5 G H z}>10^{26} \mathrm{WHz}^{-1}$ and the other having $L_{5 G H z}<10^{25} \mathrm{WHz}^{-1}$, with no quasars of intermediate luminosity. They further concluded that the fraction of quasars which are radio-loud is a function of redshift and probably optical luminosity also, and that these effects may be understood in terms of the mixing of two quasar populations with differing luminosity functions and differing amounts of cosmological evolution. (v) In both radio- 
quiet (de Bruyn \& Wilson 1978) and radio-loud (Baum \& Heckman 1989; Rawlings \& Saunders 1991) objects, there is a strong correlation between the steep-spectrum radio and the total [OIII] $\lambda 5007$ luminosities These correlations follow roughly parallel loci in the radio - $[\mathrm{OIII}]$ plane, but with the radio-loud objects a factor of $\approx 10^{4}$ more luminous at $5 \mathrm{GHz}$ than the radio-quiets for a given [OIII] luminosity. Few objects fall between the two classes (Rawlings 1993), suggesting a genuine dearth of "radio-intermediate" objects.

Observations indicate that the properties of radio-loud and radio-quiet objects are similar at infrared, optical, ultraviolet and X-ray wavelengths (e.g. Steidel \& Sargent 1991; Sanders et al. 1989; Neugebauer et al. 1986). The shapes of the local optical luminosity functions of radio-loud and radio-quiet quasars are not significantly different (Padovani 1993), although the former function is subject to large observational uncertainties. There is now considerable evidence that most of the infrared - X-ray continuum is dominated by thermal emission which, in the context of black hole accretion models, is related to the black hole mass and the ratio of the accretion rate to the Eddington accretion rate (i.e. the Eddington ratio). These similarities of the thermal properties of the two classes suggest that the distributions of black hole masses and Eddington ratios are not widely different. It is then reasonable to infer that the distinction between the radio-loud and radio-quiet classes is related to the spin of the black hole.

The hypothesis (Toomre \& Toomre 1972) that many elliptical galaxies are formed by the merging of spirals is supported by recent observations (Schweitzer 1982; Kormendy \& Djorgovski 1989). Images of radio-loud AGN show clear evidence for morphological peculiarities (e.g. tails, bridges, shells, dust lanes, second nuclei) indicative of a galaxy interaction or merger involving at least one disk galaxy (e.g. Heckman et al. 1986; Smith et al. 1986; Hutchings 1987). Such evidence for interactions/mergers is found much less frequently in radio-quiet objects (e.g. Hutchings \& Neff 1990). Thus, there is clear evidence that the radio-loud AGN phenomenon is triggered by a recent $\left(\leq 10^{9}\right.$ yrs ago $)$ interaction or merger.

The present paper presents a model which attempts to account for the dichotomy 
between the radio-loud and radio-quiet classes. We adopt the standard supermassive black hole (SMBH) model of nuclear activity (e.g. Rees 1984). In essence, the thermal emission (optical-ultraviolet continuum, at least some of the X-rays, and infrared reradiation from dust grains) is powered by accretion. On the other hand, the mechanical output of the AGN (i.e. the radio jets) is considered to be powered by the spin of the black hole (e.g. Blandford 1990 and references therein). We shall, however, assume that the normal process of accretion through an accretion disk does not lead to a rapidly spinning SMBH, because either the accretion is intermittent (and residual coupling between the hole and surrounding gas can spin down the hole between the accretion episodes) or the accretion is not confined to a single plane over the long timescales $\left(10^{8}\right.$ yrs for a $1 \mathrm{M}_{\odot} \mathrm{yr}^{-1}$ accretion rate onto a $10^{8} \mathrm{M}_{\odot}$ hole) required to spin up the hole. Rather, SMBH's acquire a significant fraction of the maximal Kerr angular momentum only through the essentially instantaneous event of coalescence with a second black hole of similar mass.

We consider mergers in a population of parent galaxies each of which contains a single, nuclear SMBH with small or zero spin. In a galaxy merger, the two nuclear SMBH's form a binary and eventually coalesce. The time scale for this coalescence is uncertain (Begelman, Blandford \& Rees 1980), but we shall assume it is very much less than the Hubble time. Qualitatively, there are three possible outcomes of such merger. Two small (say $\simeq 10^{5}$ $\mathrm{M}_{\odot}$ ) black holes of approximately equal mass will coalesce into a rapidly spinning, low mass hole. The merging of a large $\left(\right.$ say $\left.\simeq 10^{8} \mathrm{M}_{\odot}\right)$ and a small hole will result in a slowly spinning, high mass hole. Lastly, the merging of two large holes will give a rapidly spinning, high mass hole. We propose that the radio galaxy and radio quasar phenomenon is a result of the last type of merger. The mass spectrum of SMBH's in the parent population may reasonably be expected to decline towards higher mass, so most mergers will involve relatively low mass holes and will not result in a high radio luminosity object. Because of the requirement that the holes are both massive and of comparable mass, the powerful radio galaxy/quasar phenomenon is intrinsically rare, and only results from a very small fraction of galaxy mergers. 
In Section 2, we calculate the distributions of SMBH mass and jet luminosity for the merged population, while Section 3 discusses the inputs to the model in an astrophysical context. The results are compared with observations in Section 4 and discussed in Section 5. Concluding remarks and some outstanding issues and problems are given in Section 6 .

\section{THE MODEL}

We consider a parent population of galaxies each of which contains a slowly rotating or non-rotating SMBH of mass $M$ in its nucleus. The number density of SMBH's in the parent population in the mass range $M$ to $M+d M$ is $N(M) d M \mathrm{Mpc}^{-3}$ in the mass range $M_{l}$ to $M_{u}$. A fraction $g$ of these galaxies is envisaged to merge with another galaxy in a given time and the associated black holes coalesce on a timescale $\ll$ the Hubble time. We neglect the loss of mass due to gravitational radiation and also assume that there is no spatial mass segregation of the parent population of SMBH's.

\subsection{Distribution of Mass after Merging}

We use the subscript $m$ to represent parameters after merging. Two parents with masses $M_{a}$ and $M_{b}$ combine to form a daughter with mass $M_{m}$. The number density of merged SMBH's in the daughter population in the mass range $M_{m}$ to $M_{m}+d M_{m}$ is $N_{m}\left(M_{m}\right) d M_{m} \mathrm{Mpc}^{-3}$ with $N_{m}\left(M_{m}\right)$ given by

$$
\begin{aligned}
N_{m}\left(M_{m}\right) & =\frac{g}{2 N_{o}} \int_{M_{1}}^{M_{2}} N(M) N\left(M_{m}-M\right) d M \\
& =\frac{g}{N_{o}} \int_{M_{1}}^{M_{m} / 2} N(M) N\left(M_{m}-M\right) d M
\end{aligned}
$$

where $N_{o}=\int_{M_{l}}^{M_{u}} N(M) d M$ is the total number of SMBH's per Mpc ${ }^{3}$ of the parents, $M_{2}$ is the smaller of $M_{u}$ and $M_{m}-M_{l}$, and $M_{1}$ is the larger of $M_{l}$ and $M_{m}-M_{u}$.

For a power-law distribution $N(M)=K M^{-\gamma}$ ( $K$ is a normalization constant) and substituting $x=M / M_{m}$, we have

$$
\begin{aligned}
N_{m}\left(M_{m}\right) & =\frac{g K^{2}}{2 N_{o}} M_{m}^{-2 \gamma+1} \int_{x_{1}\left(M_{m}\right)}^{x_{2}\left(M_{m}\right)} x^{-\gamma}(1-x)^{-\gamma} d x \\
& =\frac{g K^{2}}{N_{o}} M_{m}^{-2 \gamma+1} \int_{x_{1}\left(M_{m}\right)}^{1 / 2} x^{-\gamma}(1-x)^{-\gamma} d x
\end{aligned}
$$


where $x_{2}$ is the smaller of $M_{u} / M_{m}$ and $\left(M_{m}-M_{l}\right) / M_{m}$, and $x_{1}$ is the larger of $M_{l} / M_{m}$ and $\left(M_{m}-M_{u}\right) / M_{m}$. The daughter distribution in the mass range $2 M_{l} \ll M_{m}<M_{u}+M_{l}$ (i.e. $x_{1}=M_{l} / M_{m} \ll 1 / 2$, and $x_{2}=1-M_{l} / M_{m} \simeq 1$ ) does not show end effects of the mass range of the parents, and in this range we have

$$
N_{m}\left(M_{m}\right)=\frac{g K^{2}}{2 N_{o}} M_{m}^{-2 \gamma+1} B(1-\gamma, 1-\gamma) \quad \text { for } \gamma<1
$$

where $B$ is the Beta function, and

$$
N_{m}\left(M_{m}\right)=g K M_{m}^{-\gamma} \quad \text { for } \gamma>1
$$

Thus the slope of the daughter mass function is identical to that of the parent if $\gamma>1$.

The integral for $N_{m}\left(M_{m}\right)$ may alternatively be expressed in terms of the mass ratio of the two merging black holes $r=M_{a} / M_{b}$

$$
N_{m}\left(M_{m}\right)=\frac{g M_{m}}{N_{o}} \int_{r_{1}\left(M_{m}\right)}^{1} N\left[\frac{M_{m} r}{(1+r)}\right] N\left[\frac{M_{m}}{(1+r)}\right] \frac{d r}{(1+r)^{2}},
$$

where $r_{1}$ is the larger of $M_{l} /\left(M_{m}-M_{l}\right)$ and $\left(M_{m}-M_{u}\right) / M_{u}$. We have taken $r<1\left(M_{a}<\right.$ $M_{b}$ ) in order to avoid double-counting. For the power-law distribution $N(M)=K M^{-\gamma}$, equation (5) becomes

$$
N_{m}\left(M_{m}\right)=\frac{g K^{2}}{N_{o}} M_{m}^{-2 \gamma+1} \int_{r_{1}\left(M_{m}\right)}^{1}\left[\frac{r}{(1+r)^{2}}\right]^{-\gamma} \frac{d r}{(1+r)^{2}}
$$

Fig. 1 is a plot of lines of constant $M_{m}$ and $r$ in the $M_{a}-M_{b}$ plane. Each value of $M_{m}$ describes a curve for which a specific range of $r$, corresponding to the range of integration in equation (5), is allowed.

\subsection{Distribution of Jet Luminosity after Merging}

The non-thermal emission is assumed to be powered by the spin energy of the merged $\mathrm{SMBH}$. Theories of the physical processes by which power is extracted from the spinning hole are still in their infancy, so our calculation of the jet power must be considered preliminary and illustrative. It is believed that energy may be extracted from a spinning black 
hole via the magnetic field by the Blandford-Znajek process (Blandford \& Znajek 1977). The electromagnetic power available from this process depends on the hole's mass and spin, and the axial magnetic field generated by external currents. Under the assumption that the magnetic energy density near the black hole is proportional to the shear stress in the accretion disk, the resulting luminosity available for extraction is conjectured (Blandford 1990) to be

$$
L_{e m}=\alpha_{H} \lambda^{2} L_{E d d} .
$$

where $\alpha_{H}$ is an unknown constant, $L_{E d d}$ is the Eddington luminosity, and $\lambda$ is the black hole spin parameter (see Blandford 1990 and references therein). In this formalism, the relationship between the "electromagnetic mass" $M_{e m}$ (defined as $M_{e m}=\lambda^{2} M_{m}$ ) and the jet luminosity $\left(L_{e m}=\alpha_{H} \frac{4 \pi G m_{p} c}{\sigma_{T}} M_{e m}\right)$ is analogous to that between $M_{m}$ and the Eddington luminosity. We shall use equation (7) to obtain the jet luminosity, while recognising the idealised nature of the expression.

We thus need to obtain the spin parameter $\lambda$ of the daughter black hole from the merging of the two parent black holes, which are assumed to possess no spin. The parameter $\lambda$ is a measure of the spin per unit mass of the daughter and so, assuming a circular orbit for the pre-merged holes, $\lambda$ is completely determined by the ratio $r$ of the masses of the parents. We may write $\lambda$ in terms of the specific angular momentum, $a$, and the gravitational radius, $m=G M / c^{2}$, of the SMBH (cf. Blandford 1990; in relativists units $[G=c=1]$, both $a$ and $m$ have units of length, so $a / m$ is dimensionless):

$$
\lambda=\frac{\frac{a}{m}}{1+\sqrt{1-\left(\frac{a}{m}\right)^{2}}} .
$$

The calculation of $\frac{a}{m}$ from the coalescence of two black holes with arbitrary mass ratio is a difficult problem in general relativity that is currently unsolved. However, the functional forms for $\lambda$ and $\frac{a}{m}$ can be constrained using physical arguments. For reasons stated in Appendix A, we have chosen the following parameterization for $\frac{a}{m}(r)$ :

$$
\frac{a}{m}(r) \approx\left(1-\frac{4 f_{1} r}{(1+r)^{2}}\right)\left[2 \sqrt{3}+(3.95-2 \sqrt{3}) \frac{4 r}{(1+r)^{2}}\right] \frac{r}{(1+r)^{2}}
$$


where $f_{1}$ is the fraction of angular momentum at the last stable circular orbit which is lost to gravitational radiation during the plunge to coalescence for equal-mass parents. For small values of $r, \lambda(r)$ has the limiting form $\sqrt{3} r$.

Sample plots of $\lambda(r)$ are shown in Fig. 2. We have experimented with other reasonable (see Appendix A) estimates of the form for $\lambda(r)$ when calculating the distribution of jet luminosities (see below), and note there are no significant effects on our results. We vary $f_{1}$ from 0.0 to 0.5 in the following calculations.

The distribution of $M_{e m}$ after merging can be written

$$
N_{m}\left(M_{e m}\right)=\frac{g}{N_{o}} \int_{r_{1}\left(\lambda, M_{e m}\right)}^{r_{2}\left(\lambda, M_{e m}\right)} N\left[\frac{r M_{e m}}{\lambda^{2}(r)(1+r)}\right] N\left[\frac{M_{e m}}{\lambda^{2}(r)(1+r)}\right] \frac{M_{e m}}{\lambda^{4}(r)(1+r)^{2}} d r .
$$

The lower limit, $r_{1}$, is determined by solving $\lambda^{2}\left(r_{1}\right)\left(1+r_{1}\right)=M_{e m} / M_{u}$. The upper limit, $r_{2}$, is either 1 or the solution of $\lambda^{2}\left(r_{2}\right)\left(1+r_{2}\right) / r_{2}=M_{e m} / M_{l}$, whichever is smaller. The changeover between these two values for $r_{2}$ occurs at $M_{e m, c r i t}=2 \lambda^{2}(1) M_{l}$. Fig. 3 shows lines of constant $M_{e m}$ and $r$ in the $M_{a}$ vs $M_{b}$ plane. As in Fig. 1, each value of $M_{e m}$ describes a curve for which a specific range of $r$, corresponding to the range of integration in equation (10), is allowed.

For a power-law parent mass function $N(M)=K M^{-\gamma}$, equation (10) becomes

$$
N_{m}\left(M_{e m}\right)=\frac{g K^{2}}{N_{o}} M_{e m}^{-2 \gamma+1} \int_{r_{1}\left(\lambda, M_{e m}\right)}^{r_{2}\left(\lambda, M_{e m}\right)} r^{-\gamma} \lambda^{4 \gamma-4}(r)(1+r)^{2 \gamma-2} d r .
$$

The integration in equation (11) can be performed analytically in limiting cases, and results are summarized in Appendix B.

\section{ESTIMATES OF IMPORTANT PARAMETERS}

In order to apply the model described in Section 2 and derive the properties of the merged ("daughter") population, we need to estimate a number of required parameters. We first describe how the parent mass function is obtained. Then, in order to guide the comparison of the model with observations (Section 4), we also estimate canonical values for $g, \alpha_{H}$, and $\epsilon$ (the efficiency of conversion of jet power to radio emission). 


\subsection{The Parent Mass Distribution}

This distribution may be estimated from the optical luminosity function (OLF) of AGN (e.g. Marshall 1985, 1986; Boyle, Shanks \& Peterson 1988) by making assumptions about the bolometric correction (i.e. the ratio of total thermal luminosity to that in the $\mathrm{B}$ band), the Eddington ratio and the activity patterns of the AGN's over cosmological timescales. In particular, Cavaliere \& Padovani $(1988,1989)$ have derived mass distributions under three assumptions concerning the activity patterns. At one extreme is a pattern (which they label 'C' for 'continuous') of literal luminosity evolution, with each object dimming continuously over the timescale ( $\approx 3 \mathrm{Gyr}$ ) for evolution of the quasar population. High redshift quasars evolve to local Seyfert 1's in a one-to-one relationship, and AGN activity is hosted by a privileged set of some $1 \%$ of bright galaxies. A second, intermediate pattern (called ' $\mathrm{R}$ ' for 'recurrent' by Cavaliere \& Padovani) is provided by discontinuous activity in a larger fraction of galaxies, consisting of short, recurrent episodes of activity. This behavior could be driven by, for example, episodes of accretion stimulated by interaction of the host galaxy with companion. In the third pattern (labelled ' $E$ ' for 'Eddington'), which is at the opposite extreme to ' $\mathrm{C}$ ', activity represents a single, short event in the lifetime of every galaxy.

In pattern 'C', $L / L_{E}$ is estimated to be $\simeq 2 \times 10^{-4} \eta_{E,-1}$ at the present epoch, where $\eta_{E,-1}$ is the efficiency of mass - energy conversion, in units of $10^{-1}$. A small fraction of galaxies (i.e. those which exhibit activity) contain very massive $\left(10^{10}-10^{12} \mathrm{M}_{\odot}\right)$ remnants. For the case of pattern ' $\mathrm{R}$ ', Cavaliere \& Padovani (1988) favor $L / L_{E} \simeq 5 \times 10^{-2} \eta_{E,-1}$. The black hole masses for both currently active and inactive galaxies cover a range $\approx 10^{7}-10^{9}$ $\mathrm{M}_{\odot}$, with currently inactive galaxies having a space density $\simeq 500$ times that of currently active ones (see Fig. 1 of Cavaliere \& Padovani 1988). For pattern 'E' in its simplest form, $\mathrm{L} / \mathrm{L}_{E}$ is taken to be $\simeq 1$ and all galaxies contain $\mathrm{SMBH}$ with masses ranging between $\simeq 10^{6}$ and $10^{10} \mathrm{M}_{\odot}$. To illustrate the application of our model, we shall use (Section 4) the Cavaliere \& Padovani mass functions for cases ' $C$ ' and ' $R$ ', derived using $L / L_{E} \simeq 10^{-4}$ and $\mathrm{L} / \mathrm{L}_{E} \simeq 5 \times 10^{-2}$, respectively. 


\subsection{The Merger Rate}

The parameter $g$ represents the fraction of SMBH's in a unit volume of space that merge in the relevant time interval. Because the merged population of interest comprises those galaxies currently active as radio-loud AGN's, this time interval is the average lifetime of the extended radio sources, which is commonly estimated to be $\tau_{R} \simeq 10^{8}$ years. The fraction of black holes which merge in this time depends on both the galaxy merger rate and the time required for the two SMBH's to coalesce. Both of these timescales are controversial. The merger rate is undoubtedly a function of both the environment and the mass ratio of the two galaxies. Schweizer $(1982,1990)$ argues that all ellipticals may have undergone at least one merger per Hubble time $\tau_{H}$. Merger rates of 1 per $10^{9}$ yrs may apply to close binary galaxies (Borne 1984) or groups (Navarro, Mosconi \& Garcia Lambas 1987). Roos (1981) estimates a merger rate of 1 per $10^{9}$ yrs when the second galaxy has $1 \%$ of the mass of the first. Heckman, Carty \& Bothun (1985) have shown that the average local galaxy density is larger (by at least a factor of $2-3$ ) for radio-loud ellipticals and lenticular galaxies than for radio-quiet galaxies of the same morphological type. Therefore, it is likely that the somewhat higher merger rates (i.e. 1 in $10^{9}$ yrs) apply to radio galaxies. The time to coalescence, $\tau_{C}$, of the SMBH's has been variously claimed to be as short as $\approx 10^{7}$ years (Ebisuzaki, Makino \& Okumura 1991) or longer than the Hubble time (e.g. Valtaoja, Valtonen \& Byrd 1989). In our model, we obviously require $\tau_{C}<<\tau_{H}$, and probably $\tau_{C} \leq 10^{9}$ yrs (so that the radio galaxy is recognisable as a recent merger). Writing $\tau_{M}=10^{9}$ yrs for the average time between mergers, we take as our canonical value $g \simeq \tau_{R} / \tau_{M} \simeq 10^{8} / 10^{9} \simeq 0.1$.

For $\tau_{R} \simeq 0.1 \tau_{M}$, it is unlikely that the merged galaxy will suffer a second merger during its lifetime as a radio source. Therefore, we do not need to consider the effect of a second black hole merger event on the mass and spin of the merged hole during the lifetime of most radio sources.

\subsection{The Electromagnetic Extraction Efficiency}


The parameter $\alpha_{H}$ is a measure of the efficiency of conversion of the spin energy of the daughter SMBH to jet power. We shall adopt $\alpha_{H}=1$ as a canonical value.

\subsection{The Efficiency of Conversion of Jet Power to Radio Emission}

Here the typically quoted numbers of $\epsilon=L_{\text {radio }} / L_{e m} \simeq 10^{-1}-10^{-2}$ may be considered canonical (see Leahy 1991, who also discusses the limitations of this form of parameterisation of the radio source energetics).

The effects of changes in these parameters on the properties of the OLF and radio luminosity function (RLF) of the merged (radio-loud) population are easily gauged. Increases in $\mathrm{N}(\mathrm{M})$ (i.e. a greater density of parent holes of a given mass) and $g$ increase the daughter number density at a given mass and luminosity. Increasing $M$ increases the optical and radio luminosities, while altering $\alpha_{H}$ and $\epsilon$ affects only the radio luminosities. In fact, the radio luminosities depend on the product $\alpha_{H} \epsilon$, not on $\alpha_{H}$ and $\epsilon$ separately.

\section{COMPARISON WITH OBSERVATIONS}

In comparing our model with the OLF and RLF of active galaxies, we have proceeded as follows.

(a) A 'parent' population is first adopted. In the case of ' $\mathrm{C}$ ' type activity, only the currently active galaxies are considered to contain SMBH's, so the appropriate parent is the radioquiet population. Because the space density of radio-louds is much less than that of radio-quiets, we can, to a good approximation, use the total (radio-quiet plus radio-loud) population as the parent. For ' $\mathrm{R}$ ' type activity, the whole galaxy population, which is dominated by currently inactive galaxies (see Fig. 1 of Cavaliere \& Padovani 1988), should be taken as the parent.

(b) To derive the parent mass distribution, we first fitted the B band OLF $\mathrm{N}_{t o t}\left(M_{B}\right)$ of the total population of AGN (Padovani 1993; Cheng et al. 1985) with a two power law 
form (cf. Marshall 1986; Boyle, Shanks \& Peterson 1988). The function chosen was:

$$
N\left(L_{B}\right)=\frac{N^{*}}{L_{B}^{*}} \times\left\{\begin{array}{ll}
\left(\frac{L_{B}}{L_{B}^{*}}\right)^{-2.2} & L<L_{B}^{*} \\
\left(\frac{L_{B}}{L_{B}^{*}}\right)^{-3.5} & L \geq L_{B}^{*}
\end{array}\right],
$$

where $N^{*}=5 \times 10^{-8} \mathrm{Mpc}^{-3}$. The luminosity function changes slope at $L_{B}^{*}=5.8 \times 10^{10}$ $\mathrm{L}_{\odot}$, which corresponds to $M_{B}^{*}=-23.65$. The data and the fitted function are plotted in Fig. 4. By adopting a value, $\mathrm{b}$, for the fraction of thermal emission which is radiated in the B band ( $b \simeq 0.03$; Blandford 1990), the total thermal luminosity corresponding to a given $M_{B}$ may be found. Given the assumed Eddington ratios $\left(L / L_{E}\right)$ adopted by Cavaliere \& Padovani (1988) and summarised above, the distribution of black hole masses in currently active galaxies is then calculated. For activity pattern ' $\mathrm{C}$ ', this distribution represents the total mass distribution of SMBH's in galaxies, and is thus assumed to represent $N(M)$. For activity pattern ' $R$ ', we must multiply the mass function by the ratio of the total number density of SMBH's (assumed to be dominated by currently inactive galaxies) to the number density of currently active SMBH's. We have used the ratio $\simeq 500$ advocated by Cavaliere \& Padovani $(1988,1989)$ to obtain $N(M)$ for pattern ' $\mathrm{R}$ '. An important issue is the relationship between the activity patterns of the radio-quiet and radio-loud populations. For ' $C$ ' type activity, we assume that a radio source turns on shortly after the SMBH's have merged and continues as a radio-loud AGN for a single period of order $10^{8}$ yrs (cf. Section 3.2). For ' $\mathrm{R}$ ' type activity, the radio-quiet and radio-loud patterns are assumed to be similar: only 1 in 500 of the merged holes is radio active at any given time and, when active, the lifetime of the activity is the canonical $10^{8}$ yrs. Physically, the inactive times would correspond to periods without gaseous accretion; such accretion is necessary both for fuelling the thermal emission and for providing the magnetic field used to tap the spin energy of the hole.

(c) The distribution of merged mass, $N_{m}\left(M_{m}\right)$, was then calculated using equation (5). In fact, the ratio $N_{m}\left(M_{m}\right) / N(M)=g$ when $M_{m}=M$ (equation 4). If we assume that the values of $b$ and $L / L_{E}$ are the same for the radio-loud and radio-quiet objects, then 
$N_{r l}\left(M_{B}\right) / N_{r q}\left(M_{B}\right)=g$, independent of $b$ and $L / L_{E}$. Here $N_{r l}\left(M_{B}\right)$ is the OLF of the radio-louds and $N_{r q}\left(M_{B}\right)$ that of the radio-quiets. By fitting our calculated $N_{r l}\left(M_{B}\right)$ to Padovani's measurements, we obtain $g=0.1$ (see Fig. 4), although the slightly higher value of $g=0.3$ may apply at high optical luminosities $\left(M_{B}<-24\right)$. For a radio galaxy lifetime of $\simeq 10^{8} \mathrm{yrs}$, the implied rate of merging is 1 in $10^{9} \mathrm{yrs}$, in excellent agreement with that obtained from other considerations in Section 3.2.

(d) Given the parent mass distribution $N(M)$ (see (b) above), the value of $g$ just obtained, and the assumed forms of $\lambda(r)$ (equations 8 and 9), we calculate $N_{m}\left(M_{e m}\right)$ using equation (10). $N\left(L_{e m}\right)$ is then found (using $L_{e m}=\alpha_{H} \frac{4 \pi G m_{p} c}{\sigma_{T}} M_{e m}$; we have taken $\alpha_{H}=1$ ), with $L_{e m}$ assumed to represent the jet luminosity. Adopting a value for the efficiency, $\epsilon$, of conversion of jet power to radio emission and a form for the synchrotron spectrum (a power law $S \propto \nu^{-\alpha}$ with $\alpha=0.75$ between $10 \mathrm{MHz}$ and $100 \mathrm{GHz}$ ), we finally obtain the RLF of radio-loud AGN in terms of $P_{20}$, the monochromatic radio power at $20 \mathrm{~cm}$. The predicted curves are plotted along with the observationally determined RLF's of radio galaxies and radio-loud quasars in Fig. 5.

\section{DISCUSSION}

We have found that our model can account for the present day relative numbers of radio-loud and radio-quiet AGN's if the merger rate is 1 in $10^{9}$ yrs. However, if such a high rate were sustained over a Hubble time and is applicable everywhere, the parents (spirals) would have long since disappeared. It is known that the galaxies hosting radio-quiet AGN's tend to be in low galaxy density environments whereas those hosting radio-loud objects are found preferentially in high density environments (e.g. Smith \& Heckman 1990). A more sophisticated calculation would allow for a range of surrounding galaxy densities and hence merger rates; radio-quiet (unmerged) objects would naturally tend to be found in low density and radio-loud (merged) in high density regions. Elliptical-spiral mergers are likely to be important in the denser regions.

The good fit to the RLF shown in Fig. 5 is obtained through mergers involving the 
low mass $\left(\mathrm{M}_{B}>-24\right)$ portion of the parent mass function (Fig. 4). In this region, the mass function of the parents is $N(M) \propto M^{-2.2}$, which implies a $\operatorname{RLF} N_{m}\left(P_{20}\right) \propto P_{20}^{-3.4}$ (equation B4), in good agreement with the RLF observed above the break near $\log P_{20}=25$ $W H z^{-1}$. Mergers involving the high mass part of the parent mass function, which follows $N(M) \propto M^{-3.5}$, are predicted (equation B4) to give $N_{m}\left(P_{20}\right) \propto P_{20}^{-6}$, a much steeper form than observed in any part of the RLF. This steep predicted form at high masses is intrinsic to the conjecture (equation 7) used to calculate the radio luminosities. The formal implication is that black hole masses in FRII radio galaxies should be similar to those in radio-quiet objects with $M_{B}>-24$, rather than those with $M_{B}<-24$.

If the parent mass distribution, $N(M)$, has a sharp cut-off at masses below those probed by the OLF (Fig. 4), the predicted RLF function for $\log P_{20}<10^{26} \mathrm{WHz}^{-1}$ (corresponding to $M_{e m}<M_{e m, \text { crit }}=2 \lambda^{2}(1) M_{l}$ ) has the form $N_{m}\left(P_{20}\right) \propto P_{20}^{0.2}$ (equation B5). This last form does not fit the data near or below the break in the RLF, suggesting there is a low luminosity turnover, rather than a sharp cut-off, in the OLF at $M_{B}>-18$. Alternatively, it would be possible to make an ad hoc modification of the form of equation (7) to allow the break in the OLF (i.e. the parent mass function) to correspond to the break in the RLF.

In the context of the current model, cosmological evolution of the radio luminosities would most naturally be ascribed to a higher black hole merger rate at earlier epochs. Such would provide density evolution, but radio luminosity evolution could also occur if $\alpha_{H}$ and/or $\epsilon$ are epoch dependent.

\section{CONCLUDING REMARKS AND FUTURE WORK}

In this paper, we have assumed that radio-loud AGN have rapidly spinning, supermassive black holes (SMBH's) in their nuclei and that the radio jets are powered by the spin energy of the holes. Contrary to the conventional view that SMBH's are spun up via gaseous accretion through a disk, we argue that such holes acquire a large spin only through a merger with another SMBH. Presumably low mass SMBH's are more common 
in galactic nuclei than high mass ones, so most mergers will involve relatively low mass SMBH's. Let us also suppose that the mass of the parent galaxy is correlated with the mass of its nuclear SMBH. Bearing in mind that only mergers between galaxies of approximately equal mass are likely to give elliptical-like remnants, we may qualitatively distinguish three types of mergers, depending on the masses of the two SMBH's (and hence parent galaxies) involved.

(i) Low mass - low mass coalescences will lead to a rapidly spinning, low mass hole in an elliptical-like galaxy. Such objects are probably those ellipticals with low luminosity, pc-scale radio cores, which are common in early-type galaxies (e.g. Sadler et al. 1994).

(ii) Low mass - high mass coalescences result in a slowly spinning, high mass hole. The merged galaxy will retain the morphological type of the more massive galaxy and such galaxies will form part of the radio-quiet population. However, these objects are expected to comprise only a small fraction of the radio-quiet population, which is dominated by the unmerged galaxies (parents).

(iii) High mass - high mass coalescences form a rapidly spinning, high mass hole. We associate these rare events with the formation of a powerful radio source.

Our idealised calculations show that both the optical and radio luminosity functions of powerful radio galaxies can be reproduced by a simple model. Merger rates of 1 per $10^{9}$ yrs are required in the regions where FRII radio galaxies form. The product of the parameters $\alpha_{H}$ (defined by $\alpha_{H}=L_{e m} / \lambda^{2}(r) L_{E d d}$, where $L_{e m}$ is the jet luminosity, $\lambda$ the black hole spin parameter, and $L_{E d d}$ is the Eddington luminosity) and $\epsilon\left(\epsilon=L_{\text {radio }} / L_{e m}\right)$ is constrained to be $\approx 5 \times 10^{-2}-10^{-4}$, depending on which pattern of evolution of AGN activity is chosen (Section 4).

In summary, our model accounts naturally for the following observations:

(1) No spiral galaxies host radio-loud AGN.

(2) Powerful radio galaxies and quasars are the products of a recent merger.

(3) Only a tiny fraction of galaxy mergers produce a radio galaxy (despite the belief that 
SMBH's are common in galactic nuclei).

(4) Radio-loud AGN are much rarer than radio-quiet AGN.

We conclude by listing a number of issues and problems which require further work.

(a) Measurements of the galaxy density and merger rate in the environment of powerful radio sources are needed. Further observational work on FRII radio galaxies is particularly desirable. It is, of course, possible that some radio galaxies are the result of the merger of an isolated binary galaxy. In that case, the density of galaxies around the radio galaxy after the merger would be a poor indicator of the relevant merger rate.

(b) Further calculations of the timescale for coalescence of two nuclear SMBH's in a galaxy merger due to stellar and gas dynamical processes are important.

(c) The controversial (e.g. Punsly \& Coroniti 1990) topic of energy extraction from spinning SMBH's deserves more study, in order to provide a firmer estimate of the jet power.

We thank R. D. Blandford, J. P. Henry, A. Illarionov, T. Jacobson, L. Kidder, M. Livio, E. Poisson, and B. C. Whitmore for valuable discussions. This work was supported in part by NASA grants NAGW-2689 and NAGW-3268. 


\section{REFERENCES}

Antonucci, R. R. J. 1993, ARAA, 31, 473

Auriemma, C., Perola, G. C., Ekers, R., Fanti, R., Lari, C., Jaffe, W. J. \& Ulrich, M. H. 1977, A\&A, 57, 41

Baum, S. A. \& Heckman, T. M. 1989, ApJ, 336, 702

Begelman, M. C., Blandford, R. D. \& Rees, M. J. 1980, Nature, 287, 307

Blandford, R. D. 1990, In Active Galactic Nuclei, ed T. J.-L. Courvoisier \& M. Mayor, Saas-Fee Advanced Course 20 (Berlin: Springer-Verlag), 161

Blandford, R. D. \& Znajek, R. L. 1977, MNRAS, 179, 433

Borne, K. D. 1984, ApJ, 287, 503

Boyle, B. J., Shanks, T. \& Peterson, B. A. 1988, MNRAS, 235, 935

de Bruyn, A. G. \& Wilson, A. S. 1978, A\&A, 64, 433

Cavaliere, A. \& Padovani, P. 1988, ApJ, 333, L33

Cavaliere, A. \& Padovani, P. 1989, ApJ, 340, L5

Cheng, F., Danese, L., de Zotti, G. \& Franceschini, A. 1985, MNRAS, 212, 857

Ebisuzaki, T., Makino, J. \& Okumura, S. K. 1991, Nature, 354, 212

Edelson, R. A. \& Malkan, M. A. 1986, ApJ, 308, 59

Elvis, M., Green, R. F., Bechtold, J., Schmidt, M., Neugebauer, G. Soifer, B. T., Matthews, K. \& Fabbiano, G. 1986, ApJ, 310, 291

Fanti, R. \& Perola, G. C. 1976, In Radio Astronomy \& Cosmology, IAU Symposium Nr. 74, ed D. L. Jauncey p171 (Dordrecht: Reidel)

Heckman, T. M., Carty, T. J. \& Bothun, G. D. 1985, ApJ, 288, 122

Heckman, T. M. et al. 1986, ApJ, 311, 526

Hutchings, J. B. 1987, ApJ, 320, 122

Hutchings, J. B., Janson, T. \& Neff, S. G. 1989, ApJ, 342, 660

Hutchings, J. B. \& Neff, S. G. 1990, AJ, 99, 1715 
Kellermann, K. I., Sramek, R. A., Schmidt, M., Shaffer, D. B., \& Green, R. 1989, AJ, 98, 1195

Kidder, L. E., Will, C. M., \& Wiseman, A. G. 1993, Physical Review D, 47, 8, 3281

Kormendy, J. \& Djorgovski, S. 1989, ARAA, 27, 235

Leahy, J. P. 1991, In Beams and Jets in Astrophysics, Ed. Philip A. Hughes, p 100 (Cambridge University Press)

Malkan, M. A. 1984, ApJ, 287, 555

Marshall, H. L. 1985, ApJ, 299, 109

Marshall, H. L. 1986, In Structure and Evolution of Active Galactic Nuclei, Eds G. Giuricin et al., p 627 (Reidel: Dordrecht)

Meurs, E. J. A., \& Wilson, A. S. 1984, A\&A, 136, 206

Miller, L., Peacock, J. A., \& Mead, A. R. G. 1990, MNRAS, 244, 207

Navarro, J. F., Mosconi, M. B., \& Garcia Lambas, D. 1987, MNRAS, 228, 501

Neugebauer, G., Miley, G. K. Soifer, B. T. \& Clegg, P. E. 1986, ApJ, 308, 815

Padovani, P. 1993, MNRAS, 263, 461

Peacock, J. A., Miller, L., \& Longair, M. S. 1986, MNRAS, 218, 265

Punsly, B. \& Coroniti, F. V. 1990, ApJ, 350, 518

Rawlings, S. \& Saunders, R. 1991, Nature, 349, 138

Rawlings, S. 1994, In The First Stromlo Symposium: The Physics of Active Galaxies, ASP Conference Series Vol. 54, Eds G. V. Bicknell, M. A. Dopita, \& P. J. Quinn, p 253 (Astronomical Society of the Pacific: San Francisco)

Rees, M. J. 1984, ARAA, 22, 471

Roos, N. 1981, A\&A 104, 218

Sadler, E. M., Slee, O. B., Reynolds, J. E. \& Ekers, R. D. 1994, In The First Stromlo Symposium: The Physics of Active Galaxies, ASP Conference Series Vol. 54, Eds G. V. Bicknell, M. A. Dopita, \& P. J. Quinn, p 335 (Astronomical Society of the Pacific: San Francisco) 
Sanders, D. B., Phinney, E. S., Neugebauer, G., Soifer, B. T. \& Matthews, K. 1989, ApJ, 347,29

Schmidt, M. 1970, ApJ, 162, 371

Schweizer, F. 1982, ApJ, 252, 455

Schweizer, F. 1990, Talk given at STScI Workshop, "The Galaxy Merger Rate"

Smith, E. P., Heckman, T. M., Bothun, G. D., Romanishin, W. R., \& Balick, B. 1986, ApJ, 306, 64

Smith, E. P. \& Heckman, T. M. 1990, ApJ, 348, 38

Steidel, C. C. \& Sargent, W. L. W. 1991, ApJ, 382, 433

Strittmatter, P. A., Hill, P., Pauliny-Toth, I. I. K., Steppe, H., \& Witzel, A. 1980, A\&A, 88, L12

Toomre, A., \& Toomre, J. 1972, ApJ, 178, 623

Valtaoja, L., Valtonen, M. J., \& Byrd, G. G. 1989, ApJ, 343, 47

Véron-Cetty, M. P. \& Woltjer, L. 1990, A\&A, 236, 69

Wall, J. V., Pearson, T. J. \& Longair, M. S. 1980, MNRAS, 193, 683

Woltjer, L. 1990, In Active Galactic Nuclei, ed T. J.-L. Courvoisier \& M. Mayor, Saas-Fee Advanced Course 20, p1 (Berlin: Springer-Verlag) 


\section{Figure Captions}

Figure 1. Contours of the merged mass $M_{m}$ (solid) and the mass ratio $r$ (dashed) in the $M_{a}-M_{b}$ plane for the case $M_{l}=10^{5} \mathrm{M}_{\odot}, M_{u}=10^{9} \mathrm{M}_{\odot}$. In order to avoid double-counting, we have chosen the upper limit of integration in $r(r<1)$ from the condition $M_{a}<M_{b}$. The dotted contour is $M_{m}=M_{u}+M_{l}$. When $M_{m}<M_{u}+M_{l}$, the lower limit of integration in equations (5) and (6) is determined from $M_{a}=M_{l}$, i.e. $r_{1}=M_{l} /\left(M_{m}-M_{l}\right)$. When $M_{m}>M_{u}+M_{l}$, the lower limit is determined from $M_{b}=M_{u}$, i.e. $r_{1}=\left(M_{m}-M_{u}\right) / M_{u}$.

Figure 2. The spin parameter $\lambda$ as a function of the mass ratio $r$ for $f_{1}=0.0-0.5$. The limiting form for small $r(\lambda(r)=\sqrt{3} r)$ is shown as a dashed line.

Figure 3. Contours of the merged electromagnetic mass $M_{e m}$ (solid and dotted) and the mass ratio $r$ (dashed) in the $M_{a}-M_{b}$ plane. The solid lines represent constant $M_{e m}$ for $\mathrm{f}_{1}$ $=0$, while the dotted represent constant $M_{e m}$ for $\mathrm{f}_{1}=0.5$. The lower limit of integration $\left(r_{1}\right)$ in equations (10) and (11) is determined from $M_{b}=M_{u}$. The upper limit $\left(r_{2}\right)$ is determined by $M_{a}=M_{b}(r=1)$ for $M_{e m}>M_{e m, c r i t}=2 \lambda^{2}(1) M_{l}$, and by $M_{a}=M_{l}$ for $M_{e m}<M_{e m, c r i t}$.

Figure 4. Optical Luminosity Functions of Active Galaxies. Filled squares represent all quasars in the Padovani (1993) sample, while filled triangles correspond to Seyfert galaxy nuclei (Cheng et al. 1985). Open squares correspond to the radio-loud quasars in Padovani's sample. The parent mass function was computed by fitting the luminosity function for the whole sample (upper line) with a two power-law form (see Section 4). The merger rate of $g=10^{-1}$ was chosen so that the predicted luminosity function for the daughter (merged) population (lower curve) agrees with the data for the radio-loud quasars (Section 4).

Figure 5. Radio Luminosity Functions of Active Galaxies for $f_{1}=0.0-0.5$. The symbols represent the observationally inferred radio luminosity functions for radio galaxies 
(Auriemma et al. 1977 - squares), radio galaxies plus radio-loud quasars (Wall, Pearson \& Longair 1980 - triangles) and radio-loud quasars (Fanti \& Perola 1976 - circles). The dashed lines represent extensions of the parent mass function beyond the range in $M_{B}$ of the Cheng et al. (1985) and Padovani (1993) samples (see Figure 4). For the 'C' type models, the plotted curves correspond to $\epsilon=10^{-4}$, while for the ' $\mathrm{R}$ ' type, $\epsilon=5 \times 10^{-2}$.

Figure A1. The spin $\frac{a}{m}$ as a function of the mass ratio $r$ for $f_{1}=0.0-0.5$. The limiting form for small $r$ is shown as a dashed line.

Figure A2. The derivative of the spin parameter $\lambda$ with respect to the mass ratio $r$ as a function of $r$ for $f_{1}=0.0-0.5$. 


\section{Appendix A: The Spin Parameter $\lambda$}

The spin parameter $\lambda$ and $\frac{a}{m}$ are related through the transformation

$$
\lambda=\frac{\frac{a}{m}}{1+\sqrt{1-\left(\frac{a}{m}\right)^{2}}} \quad \frac{a}{m}=\frac{2 \lambda}{1+\lambda^{2}} .
$$

The problem of finding the resulting spin $\frac{a}{m}$ as a function of $r$, the ratio of the masses of the two parent black holes, involves general relativistic computer codes and a solution is not yet available. A recent study by Kidder, Will \& Wiseman (1993) evolves the binary system down to the innermost stable circular orbit (ISCO) for arbitrary mass ratio. They find (see their Fig. 3) that the angular momentum per unit reduced mass $(\tilde{J})$ at ISCO is approximately linear in the parameter $\eta$ (the ratio of the reduced mass to the total

mass), rising from $\frac{\tilde{J}}{m}=2 \sqrt{3}$ for the test-mass case $(r \rightarrow 0, \eta \rightarrow 0)$ to $\frac{\tilde{J}}{m}=3.95$ in the case of equal masses $(r=1, \eta=0.25)$. The parameter $\eta$ can be written in terms of the mass ratio as $\eta=r /(1+r)^{2}$. In order to bridge the gap between $\frac{\tilde{J}}{m}$ at ISCO and $\frac{a}{m}$ after coalescence, we introduce a function $f$ (the fraction of angular momentum at ISCO that is lost in gravitational radiation during the plunge from ISCO to coalescence), which is also a function of the mass ratio $r$. Then we can write

$$
\begin{gathered}
\frac{a}{m}(r)=(1-f(r))\left(\frac{\tilde{J}}{m}\right)_{I S C O} \eta \\
\approx(1-f(r))\left[2 \sqrt{3}+(3.95-2 \sqrt{3}) \frac{4 r}{(1+r)^{2}}\right] \frac{r}{(1+r)^{2}} .
\end{gathered}
$$

We may place restrictions on $f, \frac{a}{m}$ or $\lambda$ in order to constrain the functional form $\lambda(r)$. We must have

$$
0<\frac{a}{m}(r)<1 \text { and } 0<\lambda(r)<1 \quad \text { for } \quad 0<r<1
$$

It is obvious that we must also have

$$
0<f(r)<1 \text { for } 0<r<1 \text {. }
$$

With this restriction, the requirement that $\frac{a}{m}<1$ always holds true. 
In the test-mass limit, we assume that the loss of angular momentum during the plunge is negligible $(f(0)=0)$, which yields a limiting form for $\lambda(r)$ :

$$
\lambda(r)=\frac{1}{2} \frac{a}{m}(r)=\sqrt{3} r \quad \text { as } \quad r \rightarrow 0 .
$$

One expects the spin to increase monotonically with the ratio of the masses of the parent black holes, which implies an additional requirement of

$$
\frac{d \frac{a}{m}}{d r}>0, \quad \text { or, equivalently, } \quad \frac{d \lambda}{d r}>0 \quad 0<r<1 .
$$

Various functional forms for $f(r)$ were tried. A simple linear form $f(r)=a+b r$ $(a=0, b<1)$ fails criterion (iv). Other forms (e.g. polynomial, polynomial/exponential combination) can be constructed to satisfy $(i-i v)$, but such forms require either a large number of parameters (in which case interpreting variations in a single parameter is not straightforward) or a complex functional form. A linear function in $\eta(f(r)=a+b \eta ; a=$ $0, b<4)$ does satisfy $(i-i v)$ if $b<2.1$, so we have used the single-parameter form

$$
f(r)=\frac{4 f_{1} r}{(1+r)^{2}}
$$

in our calculations (section 2.2). Here $f_{1}=b / 4=f(1)$ is the fraction of angular momentum at ISCO lost to gravitational radiation during the plunge in an equal-mass system. The resulting spin $\frac{a}{m}$ and spin parameter $\lambda$ are shown in Figs. A1 and 2, respectively, for values of $f_{1}=0.0-0.5$. For all values of $f_{1}, \frac{d \lambda}{d r}=\sqrt{3}$ at $r=0$ and $\frac{d \lambda}{d r}>0$ for all $r$ (see Fig. A2). 


\section{Appendix B: Analytic Forms for the Distribution of $M_{e m}$}

Equation (11) can be simplified in limiting cases. In the following, we have assumed $\lambda(r)=\sqrt{3} r$ for all $r$ :

$\gamma<1$ and $3 M_{l}^{2} / M_{u} \ll M_{e m} \ll M_{u}$ :

$$
N_{m}\left(M_{e m}\right)=\frac{g K^{2} 3^{2 \gamma-3}}{(1-\gamma) N_{o}}\left(\frac{1}{3 M_{u}}\right)^{\frac{3 \gamma-3}{2}} M_{e m^{2}}^{\frac{-(\gamma+1)}{2}} .
$$

$\gamma=1$ and $M_{e m, c r i t}<M_{e m} \ll M_{u}$ :

$$
N_{m}\left(M_{e m}\right)=\frac{g K^{2} 3^{2 \gamma-2}}{N_{o}} M_{e m}{ }^{-1} \ln \left(\sqrt{\frac{3 M_{u}}{M_{e m}}}\right) .
$$

$\gamma=1$ and $3 M_{l}^{2} / M_{u} \ll M_{e m}<M_{e m, c r i t}:$

$$
N_{m}\left(M_{e m}\right)=\frac{g K^{2} 3^{2 \gamma-2}}{N_{o}} M_{e m}{ }^{-1} \ln \left(\frac{1}{M_{l}} \sqrt{\frac{M_{e m} M_{u}}{3}}\right) .
$$

$\gamma>1$ and $M_{e m, c r i t}<M_{e m} \ll M_{u}$ :

$$
N_{m}\left(M_{e m}\right)=\frac{g K^{2} 3^{2 \gamma-3}}{(\gamma-1) N_{o}} M_{e m}{ }^{-2 \gamma+1} .
$$

$\gamma>1$ and $3 M_{l}^{2} / M_{u} \ll M_{e m}<M_{e m, c r i t}:$

$$
N_{m}\left(M_{e m}\right)=\frac{g K^{2} 3^{2 \gamma-3}}{(\gamma-1) N_{o}}\left(\frac{1}{3 M_{l}}\right)^{3 \gamma-3} M_{e m}^{\gamma-2} .
$$

